\title{
DISTRIBUTED MOBILE ROBOTS
}

\author{
TEJAS K PATEL AND TARIQ P SATTAR \\ Centre for Automated and Robotic NDT, London South Bank University, \\ Department of Engineering and Design, 103 Borough Road, London, SE1 OAA.
}

\author{
HERNANDO LEON RODRIGUEZ
}

Industrial Engineering Department, Faculty of Engineering, Nueva Granada Military University, Bogotá - Colombia, hernando.leon@unimilitar.edu.co

\begin{abstract}
A control scheme and a sensor system are developed to enable a group of robots to work collaboratively. The control scheme maintains a desired configuration of a group of robots while they follow the trajectory of the leader robot. One robot is a pre-defined leader and other robots in the team are followers which follow the trajectory of the leader while maintaining a desired separation distance from neighbouring robots. Simulation results are presented to show the performance of a novel control scheme. The scheme requires measurement of the distance between each robot and a pre-designated neighbour which it will follow. It also requires measurement of the angle between the orientations of the two robots. A real-world test rig is described which uses the Nintendo Wii motionplus remote controllers to obtain the necessary sensor data. The Nintendo Wii motionplus controller provides very cheap but sophisticated electronics (a 3-axis accelerometer, a 3-axis gyroscope, an Infra-Red camera and Bluetooth transmitter/receiver) that is used as a sensor system on each robot to enable sensing of the motion trajectory of the leader robot or its nearest neighbouring robot.
\end{abstract}

\section{Introduction}

Collaborative robots have been studied in recent years. The use of multiple robots has many uses including surveillance or for search and rescue during natural disasters where the use of simple cheap robots which can be deployed in large numbers would assist in the rescue of human beings by being able to search in unsafe places that are more difficult than using existing methods.

A leader-follower flocking system is reported in (1), the authors investigate a system where a few robots are leader robots "who have global knowledge (a desired trajectory), while majority of the members do not have global knowledge". The follower robots determine the flocking algorithm based on estimation of the position of the flocking centre. They also look at the possibility of followers being able to move away from the flocking group and lead to a group splitting. 
We are primarily interested in using multiple robots in the task of conducting Non-Destructive Testing (NDT) where the use of robots working as a team would speed up the inspection of large areas. For example the NDT of large steel plates used in the construction of very large infrastructure is a time consuming task. A large team of robots searching for defects with a predefined configuration would speed up the inspection of these plates.

This paper presents work being done to develop a Distributed Robots System (DRS), a group of collaborating mobile robots where individual robots (follower robots) in the group respond to rotations and movements by a predefined leader robot $(2,3)$. Each robot in the group maintains a given fixed distance relative to its neighbouring robot while following the leader's trajectory. This distance can be adjusted depending on the size of defects to be found by the robots i.e. to detect large defects the robots can be further apart while for small defects they are closer to each other. The follower robots in the group use wireless sensors to collate the information necessary about the rotations and motion of the robot designated as its nearest neighbour (which will depend on the configuration which the robots are required to maintain and thus could be in front of the robot or to its side, etc.). Each robot in the group follows the designated nearest neighbour. Thus a chain of robots is constructed leading ultimately to the leader robot.

The leader robot can be controlled remotely by an operator or be set up to move autonomously, the follower robot measures the angle of its neighbouring robot and distance between itself and its neighbouring robot to compute the trajectory of the robot, which it then tries to match. This paper is divided into the following sections. Review of the related work is presented in section 2 , followed by the configurations we have tested in section 3 , a overview of our simulation model is given in section 4 followed by simulation results in section 5 and details on how the test rig is being developed are given in section 6 followed by the conclusion in section 7 .

\section{Related Work}

In recent years collaborative robots have become an increasingly active research area due to the advantages of using multiple robots to tackle complex tasks with simple robots or to speed up the performance of tasks. An algorithm has been proposed in (4) to improve the efficiency of a leader-follower flocking robot system. The aim is to move the follower robots into formation as fast and efficiently as possible given an input from the leader robot. The robots are then required to follow the motion of the leader robot while maintaining the formation. The follower robots are claimed to get into formation in 1.5 seconds and begin following the leader robot thereafter. Our control scheme has similar goals, however we do not require the follower robots to find and get into formation with the leader robot autonomously as we put the robots into formation prior to the movement by the leader robot. 
Another leader-follower system (5) presents a strategy aiming to remove the risk of a follower robot losing the leader robot by using inter-robot communication using ad-hoc WLAN communication. Simulation results show a group of robots moving past some obstacles during which one following robot loses the group (using traditional methods). With the use of the inter-robot communication method, the follower robot re-locates the group and continues following the leader robots motion. With traditional methods of control, the distance between the robot that loses the group and the leader robot increases constantly during the simulation while the distances between the leader and other robots in the group remain under control. The use of ad-hoc inter-robot communication maintains the distances between the leader robot and all other robots in the group.

Another method for co-operative robotics (6) uses language and talking to each other with experiments in which real and simulated robots play language games to ground concepts for effective communication about their world with the use of lexicons. The authors get the robots to play several different games such as "where we are", "go to", "how far", "what direction", "where is there" and "how long". In these games the robots need to associate words with locations (lexicons) in order to build a map of their environment and complete their task.

\section{Group Configurations}

In this work we have performed simulations of four robot configurations, see Figure 1Figure 4. The configurations are a Horizontal Chain of robots, Vertical Chain of Robots, Cross Configuration and an Array Configuration. The number of robots in each configuration have been varied with the array configuration being simulated for up to 25 robots in the group.

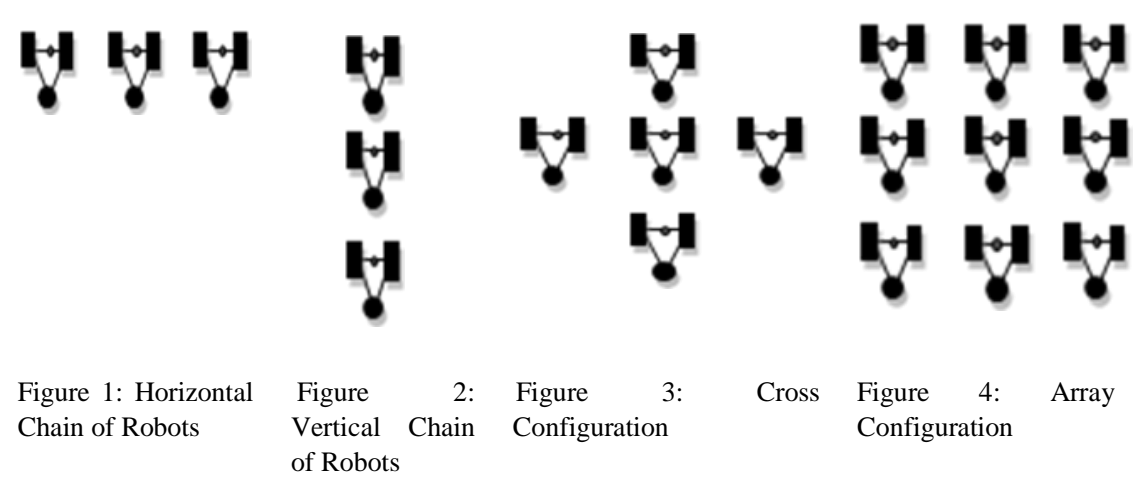




\section{Simulation Model}

Kinematics previously presented in (2) has been used for the control scheme. Each robot has two actively driven wheels actuated by two independently controlled motors and one passive wheel. A sensor system measures the distance $d$ between a robot and a designated nearest neighbour and also the angle between the orientations of the two robots. These signals are used as feedback by the digital control scheme to maintain a desired distance between the robots and a zero angle between the two orientations i.e. maintain the same heading.

We are using a multi loop multi input and single output (ML-MISO) PID controller reported in $(2,3)$ to follow the motion trajectory of the leader robot. The ML-MISO controller consists of inner and outer loops. The inner loops correct for errors between the motion of the leader robot and the follower robot (tracking error). The outer loop corrects for differences in individual robot motion dynamics to provide for greater accuracy in following the leader robot.

The ML-MISO PID controller can be fine tuned to ensure it gives the best computed value of control ' $R$ ' to be sent to the following robot, enabling it to calculate the amount it needs to move and rotate to match the movement of the lead robot. The simulation results in section 5. show the effect of the outer control loop and how it ensures that the following robots maintain the desired distance. Each robot has dynamic functions for the rotation and motion respectively, the rotation dynamic block employs a feedback loop to ensure the robot has rotated to the desired angle. The output from this block is sent to the robot trajectory block which along with the data from the motion length ' $r$ ' and previous position of the robot computes the new position of the robot at each sampling time.

The new position of the robot is then sent to the controller at each sampling time which along with the position of robot 2 and the desired distance calculates the value of ' $r$ ' that the robot 1 has moved by. The second robot uses the rotational information from the rotational dynamic block of robot 1 to rotate by the same angle and along with the information of the length ' $r$ ' from the controller the second robot is able to accurately follow changes in angle and position by the first robot.

\section{Simulation Results}

Simulation results are presented in figures Figure $5-6$. They show the accurate performance of the controller with different robot configurations (array configuration, vertical chain and a horizontal chain), with different numbers of robots in a given configuration, desired trajectories and desired distance between the robots. 


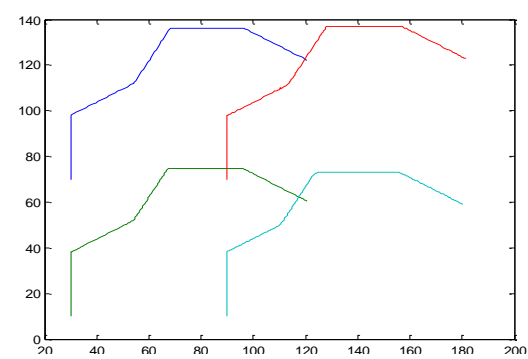

Figure 5: Four robot array configuration with distance of $20 \mathrm{~cm}$ between robots

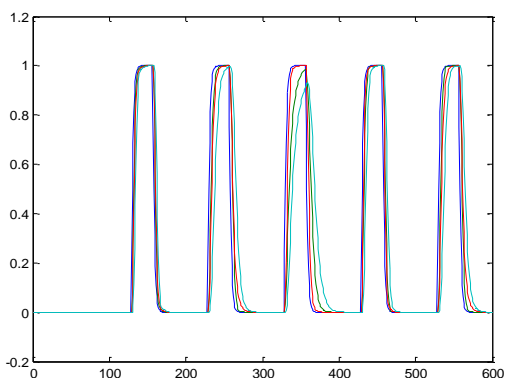

Figure 7: Motion pulse for the simulation in figure 5 with the following robots tracking the motion of the leader robot

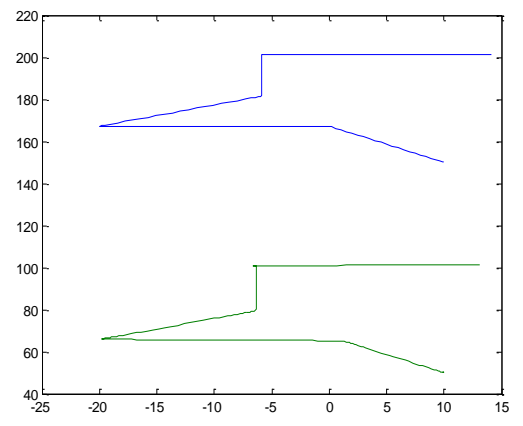

Figure 9: Two robot vertical chain with the distance set to $100 \mathrm{~cm}$

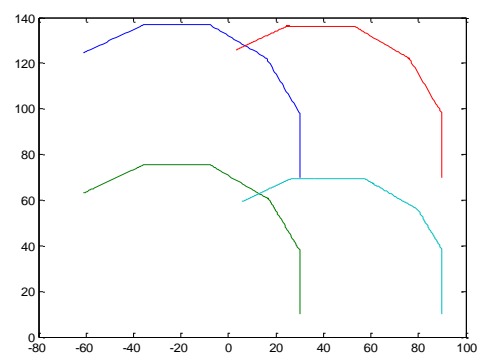

Figure 6: Four robot array configuration with distance of $20 \mathrm{~cm}$ and a different trajectory

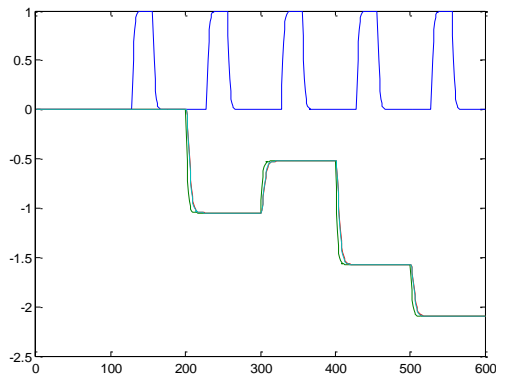

Figure 8: Angular motion graph showing the following robots tracking the rotation of the leader robot

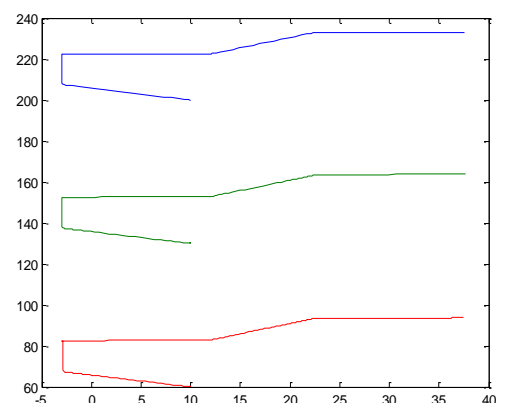

Figure 10: Three robot vertical chain with the distance set to $70 \mathrm{~cm}$ 
6

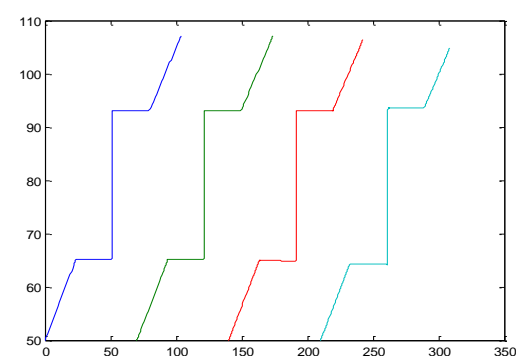

Figure 11: Four Robot Horizontal Chain

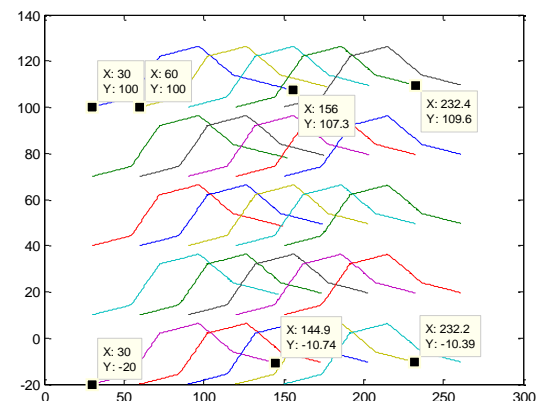

Figure 13: Motion trajectories of a 5 by 5 array configuration distributed robots

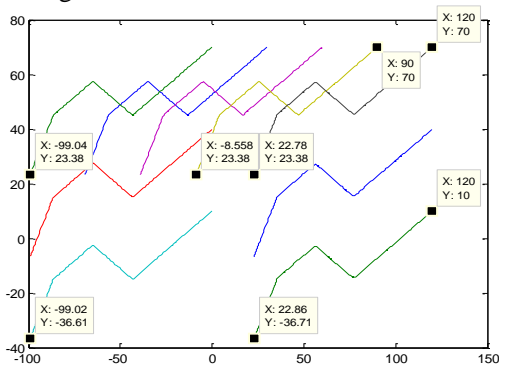

Figure 15: Motion trajectories for $\mathrm{N}$ Configuration simulation

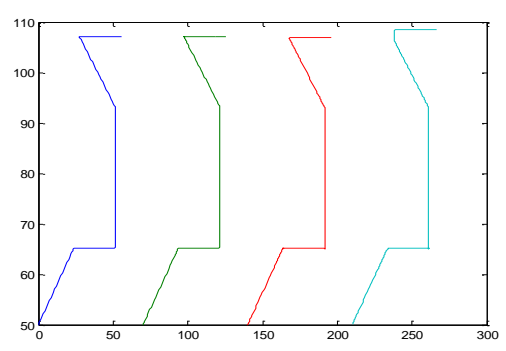

Figure 12: Four Robot Horizontal Chain

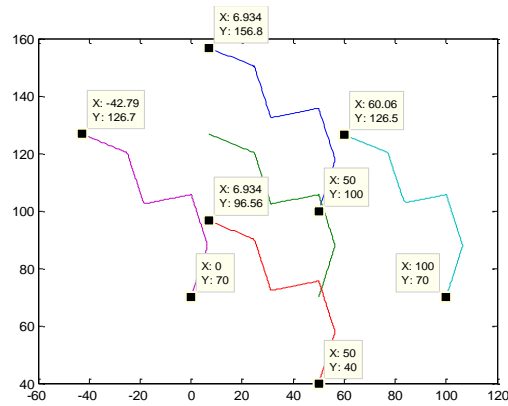

Figure 14: Motion trajectories of a cross configuration simulation

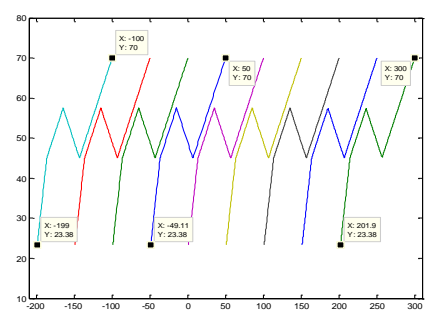

Figure 16: Motion trajectories for horizontal Nconfiguration simulation

We conducted simulations for array configurations of various sizes such as 2 by 2, 3 by 4 up to 5 by 5 for which we have presented the motion trajectory, Figure 13. Although we have not simulated array sizes greater than 5 by 5 , we are confident that the system will be able to maintain the overall configuration during the motion. The desired distance between the robots was set at $30 \mathrm{~cm}$ and from the graph in Figure 13 it can be seen that the system has largely maintained the distance while maintaining the overall configuration and having all the robots match the motion trajectory of the lead robot. 
Figure 14 shows the motion trajectories for a five robot cross configuration distributed robots system, leader robot is shown with the blue trajectory. The distance for this simulation has also been set at $30 \mathrm{~cm}$ for the vertical connection and $50 \mathrm{~cm}$ for the horizontal connection, the graph shows that again the configuration has been maintained by the system throughout the motion. The number of robots for this configuration can be increased in the four directions or a multiple cross configuration can be created by joining two cross configurations together side by side.

Figure 15 and Figure 16 show the motion trajectories for a changeable position configuration. Four robots are initially in a horizontal configuration. The first graph shows the configuration with the two end robots moved up to be vertically above the two central horizontal robots. Dynamic differences in robot dynamics and inner control loop positioning errors were incorporated into the system but the DRS managed to keep the motions of all the robots largely error free with all the robots tracking the leader robot accurately. It can be said from the results that the DRS operates correctly at any heading angle and maintains the desired distance between the robots which can be set to any value required. The number of robots in the system does not affect system operation. The errors emanating from the dynamic differences between the robots have been minimised by our control architecture resulting in a near zero error tracking of changes by other robots in the DRS.

\section{Test Rig}

A Test rig for the system is currently being developed, which involves using wireless sensors mounted on Amigobot's. Amigobot's have been developed by Adept Mobile Robots Inc. The robots are equipped with sonar sensors, gyroscopes and wi-fi communication. They have the ability to navigate autonomously in any environment using maps of the environment. We have also acquired a Pioneer 3-DX robot which has all the equipment of the Amigobot with the addition of an on-board computer and a stereoscopic camera also developed by Adept Mobile Robots (7).

Our system is being tested to verify the results obtained in simulations. Tests are being done using different configurations, distance between robots, the number of robots, (constrained to 5 for the test rig), and different motion trajectories. We are using a Nintendo Wii motion plus controller with the Wii sensor bar, the combination of the two will provide the angles and distances required for the control of our system. The Wii controller uses a high speed infrared vision camera to simultaneously track up to five infrared LED's, has 6 degrees-of-freedom using a 3-axis accelerometer, a 3-axis gyroscope, and Bluetooth technology to communicate with the Wii. It uses the standard Bluetooth HID protocol and therefore appears as a standard input to any Bluetooth host (8). 
The Wii sensor system consists of two infra-red LED clusters mounted on the leader robot with a receiver mounted on the follower; this measure's the distance between the robots and with the aid of the accelerometer provides the angle of the leader robot. The information from the Wii controller is transmitted to a remote computer via Bluetooth communication. This data is managed to compute the necessary motion trajectory by the following robots to track the leader robot. Instructions will then be transmitted to the following robot to autonomously follow the leader robot.

\section{Conclusions}

Simulations of the Distributed Robots System (DRS) show a high degree of accuracy which would enable the system to be used for Non-Destructive Testing (NDT) where the speed and accuracy matters. As the system uses multiple robots the time required to carry out of the NDT would be reduced, also if required multiple methods could be employed like ultrasound, visual inspection or eddy currents. Our simulations have been carried out using up to 25 robots; however our ML-MISO PID control with inner-outer loop control system has maintained the level of accuracy achieved while using fewer robots in the group. We are confident that we can operate the system with more than 25 robots in the group, with the accuracy still being maintained.

Future work will attempt to validate the simulation results with the test rig based on a group of five Amigo robots and the Wii gyroscope/infrared vision system.

\section{References}

1. Leader-Follower Flocking: Algorithms and Experiments. Gu, Dongbing and Wang, Zongyao. 5, s.1. : IEEE Transactions, 2009, Vol. 17. 1063-6356.

2. Distributed Mobile Robots. Sattar, Tariq, Patel, Tejas and Rodriguez, Hernando Leon. Istanbul : World Scientific, 2009. Clawar. Vol. 2009.

3. Distributed Robot Systems. Patel, Tejas K and Sattar, Tariq P. Nagoya : World Scientific, 2010.

4. Fast and Efficient Formation Flocking for a Group of Automous Mobile Robots. Xiong, Naixue, et al. 3, s.1. : IEEE, 2008, Vol. 08. 978-1-4244-1694$3 / 08$.

5. Multi-robot Formation Control Using Leader-follower for MANET. Zhang, Yi, et al. Guilin, China : IEEE, 2009. 978-1-4244-4775-6/09.

6. Robots, Communication, and Language: An Overview of the Lingodroid Project. Schulz, Ruth, et al.

7. Research Robots. Adept Mobile Robots. [Online] 2011. http://www.mobilerobots.com/ResearchRobots/ResearchRobots.aspx.

8. WiiBrew. Wiimote. WiiBrew. [Online] 3 March 2011. [Cited: 2011 March 10.] http://www.wiibrew.org/wiki/Wiimote. 